\title{
Dosimetric consequences of image guidance techniques on robust optimized intensity-modulated proton therapy for treatment of breast Cancer
}

Xiaoying Liang ${ }^{1^{*}} \mathbb{D}$, Raymond B. Mailhot Vega' ${ }^{1}$ Z Zuofeng Li ${ }^{1}$, Dandan Zheng $^{2}$, Nancy Mendenhall ${ }^{1}$ and Julie A. Bradley ${ }^{1}$

\begin{abstract}
Purpose: To investigate the consequences of residual setup error on target dose distribution using various image registration strategies for breast cancer treated with intensity-modulated proton therapy (IMPT).

Materials and methods: Among 11 post-lumpectomy patients who received IMPT, 44 dose distributions were computed. For each patient, the original plan (Plan-O) and three verification plans were calculated using different alignments: bony anatomy (VPlan-B), breast tissue (VPlan-T), and skin (VPlan-S). The target coverage were evaluated for each alignment technique. Additionally, 2 subvolumes-BreastNearSkin (1-cm rim of anterior CTV) and BreastNearCW (1-cm rim of posterior CTV) - were created to help localize CTV underdosing. Furthermore, we divided the setup error into the posture error and breast error. Patients with a large posture error and those with good posture setup but a large breast error were identified to evaluate the effect of posture error and breast error.

Results: For Plan-O, VPlan-B, VPlan-T, and VPlan-S, respectively, the median (interquartile range) breast CTV D95 was 95.7\%(94.7-96.3\%), 95.1\% (93.9-95.7\%), 95.2\% (94.8-95.6\%), and 95.2\% (94.9-95.7\%); BreastNearCW D95 was 96.9\% (95.6-97.3\%), 94.8\% (93.5-97.0\%), 95.6\% (94.8-97.0\%), 95.6\% (94.8-97.1\%); and BreastNearSkin D95 was 94.1\% (92.7$94.4 \%), 93.6 \%$ (92.2-94.5\%), 93.5\% (92.4-94.5\%), and 94.4\% (92.2-94.5\%) of the prescription dose. 4/11 patients had $\geq 1 \%$ decrease in breast CTV D95, 1 of whom developed breast edema while the other 3 all had a $>2^{\circ}$ posture error. The CTV D95 variation was within 1\% for the patients with good posture setup but $>2^{\circ}$ breast error.

Conclusion: Acceptable target coverage was achieved with all three alignment strategies. Breast tissue and skin alignment maintained the breast target coverage marginally better than bony alignment, with which the posterior CTV along the chest wall is the predominant area affected by under-dosing. For target dose distribution, posture error appears more influential than breast error.
\end{abstract}

Keywords: Breast cancer, Intensity-modulated proton therapy, Dosimetric consequences, Alignment techniques

\footnotetext{
*Correspondence: xliang@floridaproton.org

${ }^{1}$ Department of Radiation Oncology, University of Florida College of

Medicine, Jacksonville, FL, USA

Full list of author information is available at the end of the article
}

(C) The Author(s). 2020 Open Access This article is distributed under the terms of the Creative Commons Attribution 4.0 International License (http://creativecommons.org/licenses/by/4.0/), which permits unrestricted use, distribution, and reproduction in any medium, provided you give appropriate credit to the original author(s) and the source, provide a link to the Creative Commons license, and indicate if changes were made. The Creative Commons Public Domain Dedication waiver (http://creativecommons.org/publicdomain/zero/1.0/) applies to the data made available in this article, unless otherwise stated. 


\section{Introduction}

Radiotherapy plays a significant role in the treatment and cure of breast cancer. However, conventional photon-based radiotherapy, while effective, is also associated with increased risks of cardiovascular morbidity and mortality due to incidental radiation to the heart. Darby et al. [1] reported that the incidental exposure of the heart to radiation in the treatment of breast cancer increases the rate of major coronary events by $7.4 \%$ per Gy, with no apparent threshold. Due to its unique energy deposition profile, proton therapy can reduce the volume of the heart and lungs exposed to radiation, thereby potentially reducing the side-effect [2-8]. Therefore, in the past few years, there is an increasing interest in the use of proton therapy in the treatment of breast cancer $[9,10]$. While proton therapy offers significant dosimetric advantages for sparing organs at risk (OARs), it is also sensitive to setup errors. The dose distribution, which is the result of multiple pristine Bragg peaks of energy deposition, can be altered by variations in water equivalent thickness (WET) of the beam paths associated with setup uncertainty and/or organ motion.

The positioning of patients with breast cancer can be challenging due to both the large target volume and the highly mobile breast tissue. Michalski et al. [11] conducted a systematic review of the literature on inter- and intra-fraction motion in photon therapy for breast cancer. They reported an average motion below $5 \mathrm{~mm}$, but observed up to $20 \mathrm{~mm}$ in deviation for some patients. Batumalai et al. [12] reviewed the literature on setup errors in photon therapy focusing on supine breast radiotherapy using cone-beam computed tomography (CBCT) and reported up to $5.7 \mathrm{~mm}, 3.8 \mathrm{~mm}, 5.7 \mathrm{~mm}$ systematic errors and $7.3 \mathrm{~m}, 4.1 \mathrm{~mm}, 4.0 \mathrm{~mm}$ random errors in in the left-right, superior-inferior, and anteriorposterior direction, respectively.

Image-guided radiation therapy (IGRT) helps to address inter-fraction motion and improves the precision of treatment delivery. IGRT techniques have rapidly evolved for photon therapy, but in proton therapy they have remained relatively behind. Setup on bony structures on 2-dimensional images using digital radiography is currently the standard technology at proton centers. In recent years, IGRT techniques have been gradually catching up in proton therapy. CBCT has become available at some proton centers $[13,14]$ and the use of surface imaging [15] has also been reported. Despite advanced alignment techniques, it is practically impossible to have a perfect alignment on every voxel, and residual setup errors persist. To date, the consequence of the residual setup error on dose distribution for proton therapy in the treatment of breast cancer has not been reported. Many important questions remain unanswered: What is the dose error attributable to the relative motion between the breast tissue and the bony anatomy when the patient is aligned using bony structures? And which image guidance technique yields the least residual setup error so that the delivered dose is best maintained as planned? To answer these questions, we designed and conducted the current study.

\section{Methods and materials}

\section{Patient selection}

This study was approved by our institutional review board (IRB\# 201702651). This study included 11 female postlumpectomy patients consecutively treated with intensitymodulated proton therapy (IMPT). Of these, 9 patients received IMPT to the whole breast and regional lymph nodes including internal mammary nodes (IMN), axillary level I-III nodes (AxI-III), and supraclavicular nodes (SCV). 2 received IMPT to only the breast. As per standard of care at our center, all patients underwent a repeat simulation CT (thereafter referred to as verification CT) about halfway through the treatment course to evaluate breast changes (owing to breast edema, change in seroma, etc.) and setup reproducibility, and confirm stable dosimetry. The breast volume on the planning CT and the breast volume variation on the verification CT are shown in Table 1 . The treatment sites are also shown.

\section{Treatment simulation, segmentation, and planning}

Patients were simulated using a Philips Brilliance Big Bore CT (Philips Healthcare, The Netherlands) in the supine position with arms above their heads using a CIVCO breast board (CIVCO Radiotherapy, Coralville, IA, USA). Four-dimensional computed tomography (4DCT) scans were acquired, from which the averageintensity-projection CT was used for treatment planning and for generating digitally reconstructed radiography (DRR). The DRR generated from the average CT is considered a more realistic reference for daily setup given the movement of the chest wall with respiration.

The clinical target volume (CTV) structures, including breast tissue limited anteriorly $5 \mathrm{~mm}$ from the skin (CTVbreast), IMN, AxI-III, and SCV, were contoured. OARs, including the heart, left anterior descending artery (LAD), left lung, right lung, esophagus, and thyroid, were also contoured. Along the CTVs, a layer of skin structure $5 \mathrm{~mm}$ inward from the body (skin $5 \mathrm{~mm}$ rim) was contoured as well.

Treatment planning was conducted on a RayStation treatment planning system (RaySearch Laboratories, Sweden) (V6.1). The dose prescription was 50 GyRBE in 25 fractions. For each plan, 2 en-face beam angles were used. A water-equivalent 7.4-cm Lucite range shifter was used for each beam. Robust optimization was utilized on each of the CTV structure with $5 \mathrm{~mm}$ setup uncertainty and $3.5 \%$ range uncertainty. The $5 \mathrm{~mm}$ setup uncertainty 
Table 1 Patient characteristics

\begin{tabular}{llll}
\hline Patient \# & Breast tissue volume on the planning CT cm & Breast tissue volume variation on the verification CT (\%) & Treatment site \\
\hline 1 & 958.1 & 4.8 & Lt Breast only \\
2 & 372.3 & -1.1 & Lt Breast+LNs \\
3 & 742.2 & -4.5 & Lt Breast+LNs \\
4 & 858.4 & 1.8 & Lt Breast only \\
5 & 1537.7 & -4.2 & Rt Breast+LNs \\
6 & 1505.1 & -0.5 & $L t$ Breast+LNs \\
7 & 991.4 & 7.9 & Rt Breast+LNs \\
8 & 1067.0 & -4.1 & Rt Breast+LNs \\
9 & 949.2 & -6.1 & $L t$ Breast+LNs \\
10 & 1780.4 & 1.8 & $L t$ Breast+LNs \\
11 & 1361.7 & -1.7 & $L t$ Breast+LNs \\
\hline
\end{tabular}

Abbreviations: $L t$ left; Rt Right; $L N s$ lymph nodes

and $3.5 \%$ range uncertainty robust optimization parameters were based on existing literatures $[11,12,16]$ as well as our clinical experience. Both the optimization and dose computation used a Monte Carlo algorithm. The plans were normalized so that $92-95 \%$ of the target volume received $95 \%$ of the prescription dose according to the treating physician's discretion. For planning goals, ideal target coverage of CTV was V95\% $\geq 95 \%$ and D $95 \% \geq 95 \%$, but V90\% $\geq 90 \%$ and D90\% $\geq 90$ were considered acceptable.

\section{Evaluation of the impact of patient setup techniques}

To simulate patient setup during treatment [1] on bony structures using orthogonal KV imaging, [2] on soft tissue using CBCT, and [3] on skin surfaces using surface imaging, the verification $\mathrm{CT}$ was registered to the planning CT through alignment with [1] bony structures, [2] breast tissue, and [3] skin (skin $5 \mathrm{~mm}$ rim), respectively. All image registrations were performed in RayStation using automatic image registration on the specified regions of interest (ROIs) as detailed below, eliminating operator bias. The original plan was calculated on the verification $\mathrm{CT}$ and the dose distributions on the verification CT with different registration strategies were compared with the planned doses to determine the impact of the different patient setup techniques.

For each patient, 4 dose distributions were calculated:

1) Original plan (Plan-O), that is, the clinical plan used for patient treatment.

2) Verification plan using bony alignment (VPlan-B) wherein the verification $\mathrm{CT}$ was rigidly registered to the planning CT on the bony anatomy.

3) Verification plan using breast tissue alignment (VPlan-T) wherein the verification CT was rigidly registered on the breast tissue.
4) Verification plan using skin alignment (VPlan-S) wherein the verification CT was rigidly registered on the skin $5 \mathrm{~mm}$ rim.

For each verification plan, the verification dose was obtained by compute the original plan on the verification CT, using the same settings - such as pencil-beam scanning (PBS) energy layers, spot geometry and weighting, and monitor units-as those in the original plan.

The target coverage on CTVbreast was evaluated using V90, V95, D90, D95, and D99. In addition, two subvolumes-BreastNearSkin and BreastNearCWwere delineated to enable localization of specific areas of under-dosing. The BreastNearSkin and BreastNearCW were constructed by taking a $1-\mathrm{cm}$ rim of the CTVbreast volume along the skin and along the chest wall, respectively. These two subvolumes were evaluated on V90, V95, D90, and D95. The D90 and D95 on the regional lymph nodes were also evaluated. Pairwise differences in the evaluated dosimetric metrics between the original plan and each of the verification plans were evaluated using a non-parametric Wilcoxon signed rank test with $p<0.05$ taken as significant.

\section{Evaluation of setup errors}

We divided the setup error into [1] "posture error" refers to general posture setup inaccuracies, such as pitch, roll and rotation and [2] "breast error" refers to the breast rotations and shape changes with respect to the patient's bony structures. Posture errors is measured by bony anatomy difference (pitch, roll and rotation) between the verification $\mathrm{CT}$ and the reference (planning) $\mathrm{CT}$ via rigid registration using the bony structures. Breast errors can be assessed through the rotational difference between registration using the breast tissue and bony anatomy. To study the effect of posture errors and breast errors separately, we identified patients with a large posture 
error and those with good posture setup but a large breast error. The dose error of these two subgroups were evaluated. In the current study, we defined cases with $>2^{\circ}$ rotation in any direction as a large error.

\section{Results}

Target coverage with the different alignment strategies Variations in target volume delineation for breast treatment have been studied $[17,18]$ and Hurkmans et al. [18] reported a 5.5\% (standard deviation) in intraobserver variation, which aligns with our clinical experience. When re-contouring the CTVs on the verification $\mathrm{CT}$, we considered that the contouring variation would contribute $\leq 5 \%$ of the volume variation. Therefore, for cases in which the breast volume variation $>5 \%$, we further assessed these as special cases and sought a cause. We found 2 cases with $>5 \%$ breast volume variations, patients \#7 (7.9\%) and \#9 (-6.1\%), were attributed to breast edema and weight loss during the treatment, respectively. No such changes were observed in the other 9 patients. Therefore, we present our data in two groups: the first group includes the 9 patients with breast volume variations within 5\%; the second group includes the 2 special cases where edema or weight loss occurred during the treatment. For these special cases, replanning is indicated regardless of the selected alignment strategy.

\section{Target dose coverage with stable breast volume $(n=9)$}

We first evaluated the V90 and D90 on each patient with each type of alignment technique and found that our institutional goals of CTV V90 $\geq 90 \%$ and CTV D $90 \geq 90 \%$ (defined as acceptable) were met in all cases. Table 2 lists the dose statistics for CTVbreast and the two subvolume structures, BreastNearSkin and BreastNearCW. For better visualization, Fig. 1 shows the box plot on the D95 of CTVbreast, BreastNearCW, and BreastNearSkin. In general, alignment with the breast tissue and the skin marginally better preserved the CTVbreast dose compared to alignment with bony structures, with which the posterior CTV along the chest wall is the predominant area affected by under-dosing. None of the alignment strategies yielded a statistically significant difference in target dose coverage from the original plan.

For dose coverage of the regional lymph nodes, all three alignment strategies were able to meet our institutional acceptability criteria. Table 2 also lists the dose statistics on the nodal volumes. Patients \#1 and \#4 were treated on the breast only. Therefore, the data on lymph nodes includes 7 patients. The three alignment strategies yielded comparable regional lymphatics coverage and none of the alignment strategies yielded a statistically significant dose difference from the original plan.
Target dose coverage with varied breast volume $(n=2)$ Patient \#7 developed breast edema during treatment and had a $7.9 \%$ breast tissue volume increase on the verification CT. Because of the increased WET due to the edema, the CTVbreast D95 dropped by 0.8, 1.0, and $1.2 \%$ with alignment to the bony structure, breast tissue, and skin, respectively. As expected, the BreastNearCW had the largest decrease in D95: 5.8, 4.7, and 5.0\% with alignment using bony structure, breast tissue, and skin, respectively. A re-plan was performed to account for the edema owing to under-coverage near the chest wall.

Patient \#9 lost weight during treatment, resulting in a 6.1\% breast tissue volume decrease. Despite the breast tissue volume decrease, counter-intuitively, a decrease in dose coverage was observed on CTVbreast D95: 1.3, 0.6, and $0.4 \%$ decrease with alignment to bony structure, breast tissue, and skin, respectively. This patient had a $3.5^{\circ}$ posture error and the breast error was within $2^{\circ}$, indicating that large posture error is prone to underdosing the target. A re-plan was conducted for this patient due to the potential over-dose to the lung with the decreased breast volume.

\section{Posture errors and breast errors}

Table 3 shows the posture errors and the breast errors. The direction of the rotation was not indicated here; for example, we did not distinguish pitch-up from pitchdown, but chose to focus on the degree of the pitch, regardless of direction.

Among the 11 patients, 4 (patients \#1, \#4, \#9, and \#10) showed $\mathrm{a}>2^{\circ}$ posture error, mainly in the roll rotations. In addition, 6 patients $(\# 1, \# 4, \# 6, \# 8, \# 10$, and $\# 11)$ showed $\mathrm{a}>2^{\circ}$ breast error. A large posture error tends to amplify the breast error, especially for pendulous breast. Patients \#6, \#8 and \#11 had reasonable posture setup but a large breast error; therefore, they are good candidates for studying the effect of breast error alone.

Figure 2 shows the image registration of the verification CT (orange) with the planning CT (blue). Figure 2a (Patient \#4) had a counter-clock wise roll posture error so that the alignment on bony structures required a clock-wise roll correction, as illustrated in VPlan-B. Due to the counter-clockwise roll posture error, gravity created a breast tissue roll counter-clockwise, to a degree larger than the posture error alone. Therefore, a more clockwise roll correction was required when aligning with the breast tissue, as shown in VPlan-T. The alignment on skin (VPlan-S) showed an overall similar alignment as VPlan-T, but with slightly better alignment on the CTVbreast near the skin and slightly worse alignment on the CTVbreast near the chest wall. Figure $2 \mathrm{~b}$ (Patient \#11) had a good posture setup. However, due to the large $\left(1361.7 \mathrm{~cm}^{3}\right)$ and pendulous breast, the breast shape changed, requiring a counter clockwise roll to 
Table 2 Median (IQR) of the CTV Breast, BreastNearSkin, BreastNearCW, and CTV nodal coverage. The dose is displayed in percentage of the prescription dose

\begin{tabular}{|c|c|c|c|c|c|c|c|c|c|c|c|}
\hline \multirow{2}{*}{$\begin{array}{l}\text { Dosimetric } \\
\text { parameters }\end{array}$} & \multicolumn{2}{|c|}{ Plan-O } & \multicolumn{2}{|c|}{ Vplan-B } & \multicolumn{2}{|c|}{ VPlan-T } & \multicolumn{2}{|c|}{ VPlan-S } & \multicolumn{3}{|l|}{$P$ values } \\
\hline & Med & IQR & Med & $\mathrm{IQR}$ & Med & IQR & Med & IQR & $\begin{array}{l}\text { Plan-O vs. Vplan- } \\
\text { B }\end{array}$ & $\begin{array}{l}\text { Plan-O vs. Vplan- } \\
\text { T }\end{array}$ & $\begin{array}{l}\text { Plan-O vs. Vplan- } \\
\text { S }\end{array}$ \\
\hline CTVbreast V90 (\%) & 99.7 & $99.3-99.8$ & 98.8 & $\begin{array}{l}98.7- \\
99.6\end{array}$ & 99.6 & $\begin{array}{l}98.6- \\
99.6\end{array}$ & 99.6 & $\begin{array}{l}98.6- \\
99.8\end{array}$ & 0.22 & 0.34 & 0.60 \\
\hline CTVbreast V95 (\%) & 96.4 & $\begin{array}{l}93.32- \\
97.33\end{array}$ & 95.1 & $\begin{array}{l}91.7- \\
96.3\end{array}$ & 95.2 & $\begin{array}{l}94.1- \\
97.0\end{array}$ & 95.3 & $\begin{array}{l}94.7- \\
97.0\end{array}$ & 0.55 & 0.60 & 0.67 \\
\hline CTVbreast D90 (\%) & 96.5 & $95.4-97.5$ & 96.3 & $\begin{array}{l}95.3- \\
97.5\end{array}$ & 96.4 & $\begin{array}{l}95.6- \\
97.3\end{array}$ & 96.4 & $\begin{array}{l}95.7- \\
97.5\end{array}$ & 0.75 & 0.80 & 0.93 \\
\hline CTVbreast D95 (\%) & 95.7 & $94.7-96.3$ & 95.1 & $\begin{array}{l}93.9- \\
95.7\end{array}$ & 95.2 & $\begin{array}{l}94.8- \\
95.6\end{array}$ & 95.2 & $\begin{array}{l}94.9- \\
95.7\end{array}$ & 0.22 & 0.45 & 0.55 \\
\hline CTV Breast D99 (\%) & 92.6 & $90.8-92.8$ & 89.2 & $\begin{array}{l}88.6- \\
91.6\end{array}$ & 91.8 & $\begin{array}{l}88.5- \\
92.7\end{array}$ & 91.8 & $\begin{array}{l}88.5- \\
93.0\end{array}$ & 0.60 & 0.55 & 0.62 \\
\hline BreastNearCW V90 (\%) & 99.6 & $99.0-99.9$ & 99.0 & $97.3-100$ & 98.9 & $98.3-100$ & 99.1 & $97.8-100$ & 0.59 & 0.47 & 0.62 \\
\hline BreastNearCW V95 (\%) & 98.3 & $97.7-99.5$ & 94.6 & $\begin{array}{l}93.4- \\
99.7\end{array}$ & 97.0 & $\begin{array}{l}94.6- \\
99.7\end{array}$ & 97.1 & $\begin{array}{l}94.7- \\
99.8\end{array}$ & 0.49 & 0.67 & 0.80 \\
\hline $\begin{array}{l}\text { BreastNearCW D90 } \\
(\%)\end{array}$ & 97.4 & $96.0-97.8$ & 96.5 & $\begin{array}{l}95.9- \\
97.6\end{array}$ & 97.3 & $\begin{array}{l}95.9- \\
97.7\end{array}$ & 97.5 & $\begin{array}{l}95.9- \\
97.8\end{array}$ & 0.34 & 0.39 & 0.86 \\
\hline $\begin{array}{l}\text { BreastNearCW D95 } \\
(\%)\end{array}$ & 96.9 & $95.6-97.3$ & 94.8 & $\begin{array}{l}93.5- \\
97.0\end{array}$ & 95.6 & $\begin{array}{l}94.8- \\
97.0\end{array}$ & 95.6 & $\begin{array}{l}94.8- \\
97.1\end{array}$ & 0.18 & 0.27 & 0.45 \\
\hline $\begin{array}{l}\text { BreastNearSkin V90 } \\
\text { (\%) }\end{array}$ & 99.4 & $98.5-99.5$ & 98.7 & $\begin{array}{l}98.3- \\
99.5\end{array}$ & 98.9 & $\begin{array}{l}97.2- \\
99.6\end{array}$ & 99.1 & $\begin{array}{l}97.2- \\
99.6\end{array}$ & 0.49 & 0.95 & 0.84 \\
\hline $\begin{array}{l}\text { BreastNearSkin V95 } \\
(\%)\end{array}$ & 91.0 & 84.9-93.1 & 91.3 & $\begin{array}{l}83.5- \\
93.7\end{array}$ & 92.3 & $\begin{array}{l}86.0- \\
93.3\end{array}$ & 92.8 & $\begin{array}{l}85.9- \\
93.4\end{array}$ & 1.00 & 0.93 & 0.93 \\
\hline $\begin{array}{l}\text { BreastNearSkin D90 } \\
(\%)\end{array}$ & 95.5 & $94.1-96.0$ & 95.2 & $\begin{array}{l}93.6- \\
96.4\end{array}$ & 95.5 & $\begin{array}{l}94.2- \\
95.7\end{array}$ & 95.2 & $\begin{array}{l}94.2- \\
96.0\end{array}$ & 1.00 & 0.81 & 0.98 \\
\hline $\begin{array}{l}\text { BreastNearSkin D95 } \\
(\%)\end{array}$ & 94.1 & $92.7-94.4$ & 93.6 & $\begin{array}{l}92.2- \\
94.5\end{array}$ & 93.5 & $\begin{array}{l}92.4- \\
94.5\end{array}$ & 94.4 & $\begin{array}{l}92.2- \\
94.5\end{array}$ & 0.62 & 0.93 & 0.85 \\
\hline IMN D90 (\%) & 96.8 & $96.6-97.4$ & 96.5 & $\begin{array}{l}95.4- \\
97.2\end{array}$ & 96.6 & $\begin{array}{l}95.0- \\
97.1\end{array}$ & 96.5 & $\begin{array}{l}94.8- \\
96.9\end{array}$ & 0.40 & 0.54 & 0.32 \\
\hline IMN D95 (\%) & 96.1 & $95.8-96.7$ & 95.7 & $\begin{array}{l}94.6- \\
96.4\end{array}$ & 95.6 & $\begin{array}{l}94.0- \\
96.6\end{array}$ & 95.6 & $\begin{array}{l}93.5- \\
96.3\end{array}$ & 0.48 & 0.48 & 0.26 \\
\hline AXI D90 (\%) & 96.6 & $95.0-98.1$ & 96.5 & $\begin{array}{l}94.9- \\
98.0\end{array}$ & 96.5 & $\begin{array}{l}94.9- \\
98.0\end{array}$ & 96.5 & $\begin{array}{l}94.9- \\
97.8\end{array}$ & 0.71 & 0.80 & 0.93 \\
\hline AXI D95 (\%) & 96.2 & $94.2-97.8$ & 96.1 & $\begin{array}{l}94.1- \\
97.7\end{array}$ & 96.2 & $\begin{array}{l}94.1- \\
97.7\end{array}$ & 96.2 & $\begin{array}{l}94.1- \\
97.4\end{array}$ & 0.64 & 0.80 & 0.80 \\
\hline AXII D90 (\%) & 96.0 & $93.4-97.3$ & 95.9 & $\begin{array}{l}93.1- \\
97.3\end{array}$ & 96.0 & $\begin{array}{l}93.2- \\
97.3\end{array}$ & 96.0 & $\begin{array}{l}93.3- \\
97.1\end{array}$ & 0.80 & 0.90 & 0.98 \\
\hline AXII D95 (\%) & 95.6 & $92.3-96.9$ & 95.4 & $\begin{array}{l}92.6- \\
96.9\end{array}$ & 95.5 & $\begin{array}{l}92.8- \\
96.9\end{array}$ & 95.5 & $\begin{array}{l}92.8- \\
96.5\end{array}$ & 0.90 & 0.90 & 0.90 \\
\hline AXIII D90 (\%) & 96.4 & $93.6-97.1$ & 96.3 & $\begin{array}{l}93.7- \\
97.0\end{array}$ & 96.1 & $\begin{array}{l}94.0- \\
97.0\end{array}$ & 96.2 & $\begin{array}{l}93.8- \\
97.0\end{array}$ & 0.80 & 0.71 & 0.90 \\
\hline AXIII D95 (\%) & 95.9 & $92.9-96.7$ & 95.8 & $\begin{array}{l}93.1- \\
96.6\end{array}$ & 95.6 & $\begin{array}{l}93.1- \\
96.5\end{array}$ & 95.6 & $\begin{array}{l}93.2- \\
96.5\end{array}$ & 0.80 & 0.83 & 0.90 \\
\hline SCV D90 (\%) & 96.9 & $93.6-97.5$ & 96.7 & $\begin{array}{l}93.9- \\
97.3\end{array}$ & 96.6 & $\begin{array}{l}94.1- \\
97.3\end{array}$ & 96.7 & $\begin{array}{l}94.2- \\
97.3\end{array}$ & 0.78 & 0.93 & 0.74 \\
\hline SCV D95 (\%) & 96.5 & $92.5-96.9$ & 96.3 & $\begin{array}{l}93.1- \\
96.7\end{array}$ & 96.1 & $\begin{array}{l}93.2- \\
96.7\end{array}$ & 96.3 & $\begin{array}{l}93.3- \\
96.7\end{array}$ & 0.80 & 0.90 & 0.80 \\
\hline
\end{tabular}

Abbreviation: Med median; IQR interquartile range; BreastNearSkin, 1-cm rim of anterior CTV; BreastNearCW, 1-cm rim of posterior CTV

align the breast tissue (VPlan-T) and the skin (VPlan-S). The image registration for the patient who developed edema during treatment is shown in Fig. 2c (Patient \#7); an increased WET along the beam path was observed.
To study the effect of posture errors and breast errors, we examined the cases in which the CTVbreast D95 decreased by $\geq 1 \%$ of the prescription dose. In total, 4 of the 11 patients experienced $\geq 1 \%$ decrease in CTVbreast D95: 

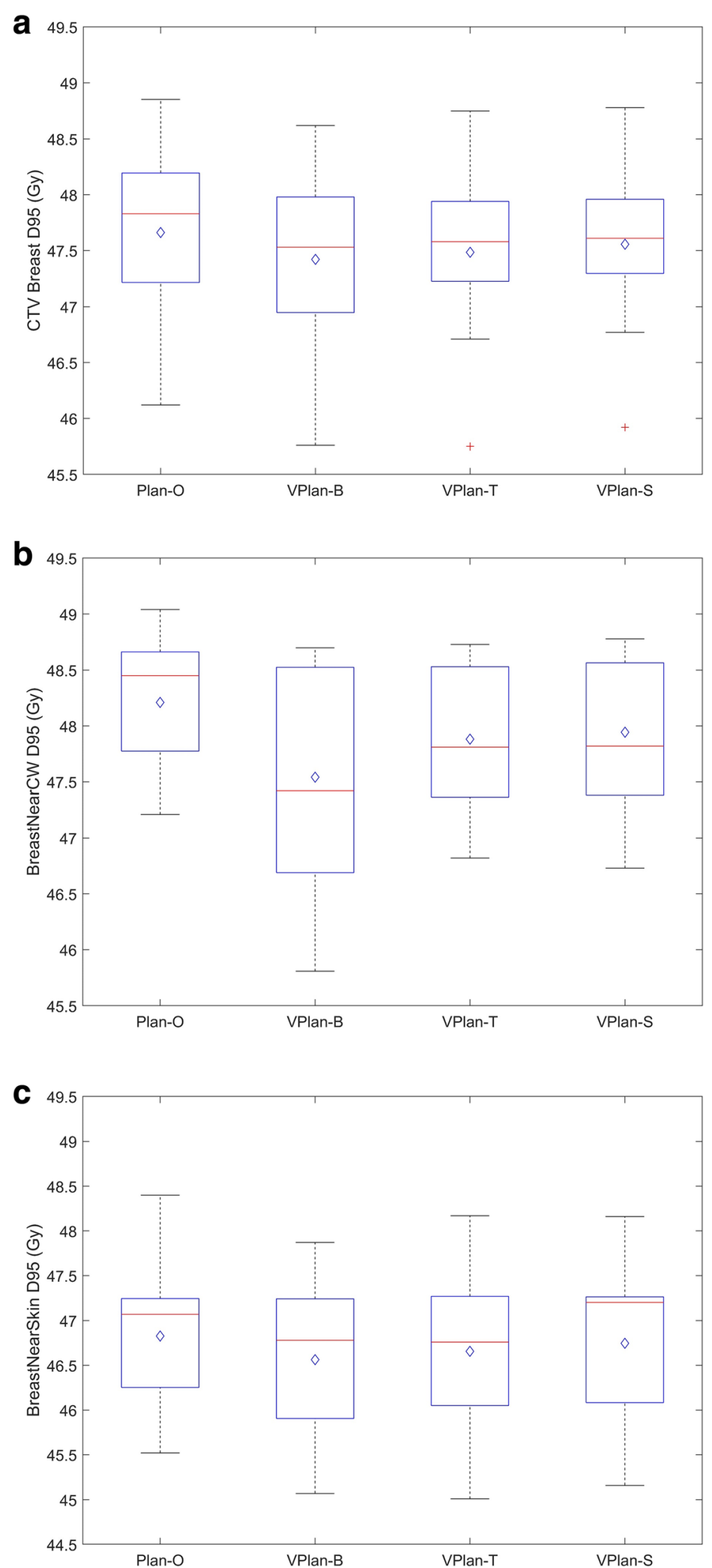

Fig. 1 Box plot of CTVbreast D95, BreastNearCW D95, and BreastNearSkin D95. The red line inside the box represents the median value and the blue diamond represents the mean value 
Table 3 Posture errors and breast errors of the 11 studied patients

\begin{tabular}{|c|c|c|c|c|c|c|}
\hline \multirow{2}{*}{$\begin{array}{l}\text { Patient } \\
\#\end{array}$} & \multicolumn{3}{|c|}{ Posture errors } & \multicolumn{3}{|c|}{ Breast errors } \\
\hline & $\overline{\text { Pitch }\left({ }^{\circ}\right)}$ & Roll $\left({ }^{\circ}\right)$ & Yaw $\left({ }^{\circ}\right)$ & $\overline{\text { Pitch }\left({ }^{\circ}\right)}$ & Roll $\left({ }^{\circ}\right)$ & $\overline{\text { Yaw }\left({ }^{\circ}\right)}$ \\
\hline 1 & 0.5 & 6.3 & 1.5 & 1.2 & 0.8 & 3.0 \\
\hline 2 & 0.5 & 0.2 & 1.1 & 0.6 & 0.2 & 1.4 \\
\hline 3 & 0.1 & 0.2 & 0.2 & 0.6 & 0.7 & 0.5 \\
\hline 4 & 0 & 2.1 & 1.3 & 0.7 & 2.8 & 1.8 \\
\hline 5 & 1.4 & 0.1 & 0.3 & 0 & 1.0 & 0.1 \\
\hline 6 & 0.2 & 1.9 & 1.2 & 0.5 & 2.6 & 0.5 \\
\hline 7 & 0.4 & 0.2 & 0.7 & 0.6 & 1.6 & 0.1 \\
\hline 8 & 0.8 & 1.0 & 1.9 & 1.1 & 1.4 & 2.4 \\
\hline 9 & 1.6 & 3.5 & 0.6 & 0 & 1.8 & 0.9 \\
\hline 10 & 2.1 & 2.2 & 0.6 & 0.5 & 2.2 & 1.0 \\
\hline 11 & 0 & 0 & 0 & 0.8 & 4.0 & 0.7 \\
\hline
\end{tabular}

Patients \#1 (1.7\% with bony alignment), \#4 (1.1\% with both breast tissue and skin alignments), \#7 (1.0\% with breast tissue and $1.2 \%$ with skin alignments), and \#9 (1.3\% with bony alignment). As Patient \#7 developed breast edema, which caused a WET increase and consequently target coverage loss, we removed her from this particular analysis. Although Patient \#9 was also a special case with weight loss, unlike Patient \#7, Patient \#9 had a breast tissue volume decrease and in principle WET decrease, and was therefore included in this analysis.

We investigated the impact of the breast error by identifying cases that had $<2^{\circ}$ setup error but $>2^{\circ}$ breast error (patients \#6, \#8 and \#11). These three cases represent scenarios in which the patient had a reasonable general posture setup, but large breast error. All of these patients had relative large breast volumes (1505.1, 1067.0, and $1361.7 \mathrm{~cm}^{3}$ ), increasing the likelihood of a change in breast shape due to the mobility of the breast tissue. However, none of these patients demonstrated a $\geq 1 \%$ decrease in CTVbreast D95 with any alignment strategies, indicating that, with our method of robust optimization, the target coverage is well maintained (within $1 \%$ variation) with any type of alignment (bony, breast tissue, skin) even for cases with large breast errors, as long as the patient's posture setup is good.

We also analyzed the cases $(\# 1, \# 4, \# 9$, and \#10) in which the posture error was large $\left(>2^{\circ}\right)$. Among these 4 cases, 3 demonstrated $\mathrm{a} \geq 1 \%$ decrease in CTVbreast D95
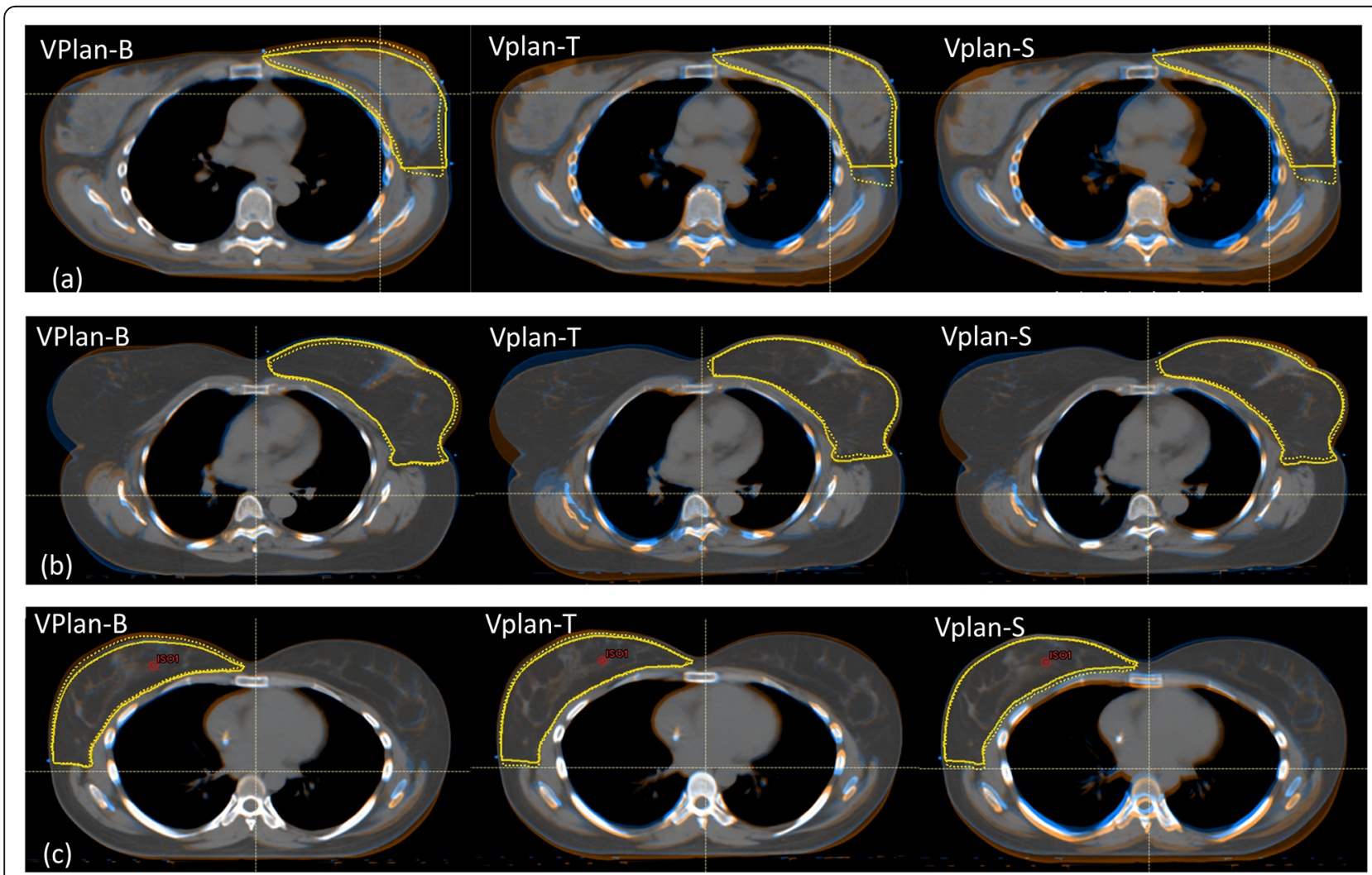

Fig. 2 Example of image registration between the verification scan (orange) and the original planning scan (blue) for (a) Patient \#4, who had a large posture error and breast error, (b) Patient \#11, who had good posture setup but a large breast error, and (c) Patient \#7, who developed breast edema during the treatment. The blue or orange color only appears in regions of misalignment. The solid yellow contour represents the CTVbreast contour on the original scan and the dashed yellow contour represents the CTVbreast on the verification scan 
with $\geq 1$ of the alignment strategies. This, together with the fact that the patients who experienced $a \geq 1 \%$ decrease in CTVbreast D95 all had a large posture error (with the exception of the breast edema case), indicate that a large posture error tend to have a higher likelihood of decrease the target coverage. Alignment with the breast tissue or with the skin does not guarantee $<1 \%$ CTV D95 variation for cases with large posture errors.

\section{Discussion}

Setup for breast patients is challenging and the dosimetric consequences for photon therapy have been wellstudied [19-23], but comparable data for proton therapy are scarce. Due to the distinctly different physical properties of photons and protons, and their different treatment planning techniques, experiences with photon therapy cannot be directly translated and applied to proton therapy. The maintenance of target coverage for patients with breast cancer in the setting of inter-fraction motion has not yet been well-studied for proton therapy. The optimal approach for setup breast patients undergoing treatment with proton therapy has not been established. Through this study, we sought to determine [1] the dosimetric consequence of different alignment techniques and [2] the optimal patient setup strategy for breast patients treated with proton therapy.

We studied 11 patients with a median CTVbreast volume of $991.4 \mathrm{~cm}^{3}$ (range, $372.3-1780.4 \mathrm{~cm}^{3}$ ) and investigated the dosimetric impact of different alignment techniques on the target coverage for patients with breast cancer undergoing treatment using IMPT. Ontreatment verification CT were registered to the planning CT using different alignment techniques: bony structure to simulate setup using orthogonal KV images, breast tissue to simulate setup using CBCT, and skin to simulate setup using surface imaging. Our study showed that our treatment planning technique is robust; acceptable target coverage was maintained using any of these three alignment strategies. Due to the relative motion between the bony structure and breast tissue, we found that the CTV along the chest wall is most susceptible to underdosing when bony structures are used for alignment. Therefore, for patients with a high-risk area near the chest wall, particular attention to the setup error is critical. In this study, we divided the setup error into posture error and breast error to investigate which error would have a greater impact on target dose coverage. We found that the posture error had a greater effect than the breast error. Patients who have a pendulous breast tend to demonstrate larger breast error, as the reproducibility of the breast shape and position is more difficult due to the mobile nature of the breast. Clinically, a smaller breast tends to flatten along the chest wall with the patient in a supine, arms up position, while a larger breast that remains pendulous in this position retains breast tissue that is can be very mobile. Larger breast size has been associated with increased acute toxicity such as breast edema [24]. Despite this tendency for higher breast error, the target coverage is well maintained (CTV D95 within 1\% variation) as long as the patient's posture setup is accurate $\left(<2^{\circ}\right.$ setup error). However, if the patient had a large $\left(>2^{\circ}\right)$ posture error, CTV D95 commonly decreased by $\geq 1 \%$, and alignment with the breast tissue or the skin was not always sufficient to overcome the posture error, resulting in decreased target coverage. The patient's body is not rigid; when the patient has a large posture error, 6D couch corrections can only be a compromise. The larger posture error in the initial setup, the more residual posture errors after couch corrections and the more dose errors will occur. Furthermore, the posture error may exacerbate the breast error, as seen in Fig. 2a, as the mobile breast tissue can align differently based on patient posture. Therefore, if $>2^{\circ}$ posture error is identified on initial patient setup for daily treatment, we recommend correction of the posture by reset up the patient and repeat imaging until a posture error $<2^{\circ}$ is achieved.

At our center, the current standard practice of breast patient setup is to use the orthogonal KV images to align with the bony anatomy. Therefore, for treatment planning, we account for the relative motion between the bony and breast tissue through robust optimization with a 5-mm setup uncertainty. It is our expectation that an acceptable dose coverage can be achieved with bony alignment. Due to the robustness of our plan, no apparent CTV dose coverage difference was observed among the three types of alignment strategies. For future research, it would be interesting to investigate whether [1] it is acceptable to robust optimize the plan with a tighter setup uncertainty if CBCT is used for daily setup, and [2] a larger difference can be observed for robust optimized plans using a tighter setup uncertainty.

In the present study, we used different alignment methods for the verification CT to mimic different IGRT techniques. The image registration was conducted in RayStation using automatic registration on selected ROIs. We have to note that the image registration algorithms used in RayStation may differ from those used in the imaging system for patient setup and treatment delivery. The simulated surface alignment using the skin $5 \mathrm{~mm}$ rim may be especially different than the true optical-based surface alignment. Furthermore, others [25] have shown that setup accuracy for surface imaging is sensitive to the ROI selection. Therefore, it is essential to evaluate the limitations of a particular image guidance system and identify the differences in image registration algorithms before directly translating the current study results to clinical patient setups. 


\section{Conclusion}

A planning technique that utilizes robust optimization on CTVs with a 5-mm setup uncertainty and $3.5 \%$ range uncertainty successfully maintains acceptable target coverage with all alignment strategies (bony anatomy, breast tissue, and skin). Breast tissue and skin alignment maintained the breast target coverage marginally better than bony alignment. When aligning with bony structure, the CTV along the chest wall is the predominant area affected by under-dosing. The posture error is more influential to the target dose distribution than the breast error.

\section{Acknowledgements}

We would like to thank Jessica Kirwan and Christopher Stich for editorial assistance.

\section{Authors' contribution}

$X L$, JB, RM developed the concept, conducted the study with the help from all other authors. $\mathrm{XL}$ carried out the date collection. $\mathrm{XL}, \mathrm{DZ}$, JB drafted the manuscript. All the authors provided clinical expertise and participated in writing the manuscript. All authors read and approved the final manuscript.

\section{Funding}

None.

\section{Availability of data and materials}

The datasets used and/or analyzed during the current study are available from the corresponding author on reasonable request.

\section{Ethics approval and consent to participate}

The study was approved by the intuitional review board (IRB201702651).

\section{Consent for publication}

Not applicable.

\section{Competing interests}

The authors received no specific funding for this work. JB and RM- travel grant from lon beam application. The other authors declare that they have no competing interests.

\section{Author details}

${ }^{1}$ Department of Radiation Oncology, University of Florida College of Medicine, Jacksonville, FL, USA. ${ }^{2}$ Department of Radiation Oncology, University of Nebraska Medical Center, Omaha, NE, USA.

Received: 23 December 2019 Accepted: 17 February 2020

Published online: 27 February 2020

\section{References}

1. Darby SC, Ewertz M, McGale P, et al. Risk of ischemic heart disease in women after radiotherapy for breast cancer. N Engl J Med. 2013;368:987-98.

2. Ares C, Khan S, Macartain AM, et al. Postoperative proton radiotherapy for localized and locoregional breast cancer: potential for clinically relevant improvements? Int J Radiat Oncol Biol Phys. 2010:76:685-97.

3. MacDonald SM, Patel SA, Hickey S, et al. Proton therapy for breast cancer after mastectomy: early outcomes of a prospective clinical trial. Int J Radiat Oncol Biol Phys. 2013;86:484-90.

4. Bradley JA, Dagan R, Ho MW, et al. Initial report of a prospective Dosimetric and clinical feasibility trial demonstrates the potential of protons to increase the therapeutic ratio in breast Cancer compared with photons. Int J Radiat Oncol Biol Phys. 2016;95:411-21.

5. Braunstein $L Z$, Cahlon O. Potential morbidity reduction with proton radiation therapy for breast Cancer. Semin Radiat Oncol. 2018;28:138-49.

6. Xu N, Ho MW, Li Z, et al. Can proton therapy improve the therapeutic ratio in breast cancer patients at risk for nodal disease? Am J Clin Oncol. 2014;37: 568-74.
7. Cuaron JJ, Chon B, Tsai H, et al. Early toxicity in patients treated with postoperative proton therapy for locally advanced breast cancer. Int J Radiat Oncol Biol Phys. 2015;92:284-91.

8. Fagundes $M$, Hug EB, Pankuch $M$, et al. Proton therapy for local-regionally, advanced breast Cancer maximizes cardiac sparing. Int J Part Ther. 2015;1: 827-44.

9. Kammerer E, Guevelou JL, Chaikh A, et al. Proton therapy for locally advanced breast cancer: a systematic review of the literature. Cancer Treat Rev. 2018;63:19-27.

10. Hug EB. Proton therapy for primary breast Cancer. Breast Care (Basel). 2018; 13:168-72.

11. Michalski A, Atyeo J, Cox J, et al. Inter- and intra-fraction motion during radiation therapy to the whole breast in the supine position: a systematic review. J Med Imaging Radiat Oncol. 2012;56:499-509.

12. Batumalai $V$, Holloway L, Delaney GP. A review of setup error in supine breast radiotherapy using cone-beam computed tomography. Med Dosim. 2016:41:225-9.

13. Veiga $C$, Janssens $G$, Teng $C L$, et al. First clinical investigation of cone beam computed tomography and deformable registration for adaptive proton therapy for lung Cancer. Int J Radiat Oncol Biol Phys. 2016;95:549-59.

14. Wang $P$, Yin $L$, Zhang $Y$, et al. Quantitative assessment of anatomical change using a virtual proton depth radiograph for adaptive head and neck proton therapy. J Appl Clin Med Phys. 2016;17:427-40.

15. Batin E, Depauw N, MacDonald S, et al. Can surface imaging improve the patient setup for proton postmastectomy chest wall irradiation? Pract Radiat Oncol. 2016:6:e235-41.

16. Paganetti $\mathrm{H}$. Range uncertainties in proton therapy and the role of Monte Carlo simulations. Phys Med Biol. 2012;57(11):R99-117.

17. Vinod SK, Jameson MG, Min M, et al. Uncertainties in volume delineation in radiation oncology: a systematic review and recommendations for future studies. Radiother Oncol. 2016:121:169-79.

18. Hurkmans $\mathrm{CW}$, Borger $\mathrm{JH}$, Pieters BR, et al. Variability in target volume delineation on CT scans of the breast. Int J Radiat Oncol Biol Phys. 2001;50: 1366-72.

19. Hector $\mathrm{CL}$, Webb S, Evans PM. The dosimetric consequences of interfractional patient movement on conventional and intensity-modulated breast radiotherapy treatments. Radiother Oncol. 2000:54:57-64.

20. Baroni G, Garibaldi C, Scabini M, et al. Dosimetric effects within target and organs at risk of interfractional patient mispositioning in left breast cancer radiotherapy. Int J Radiat Oncol Biol Phys. 2004;59:861-71.

21. Harron EC, McCallum HM, Lambert EL, et al. Dosimetric effects of setup uncertainties on breast treatment delivery. Med Dosim. 2008;33:293-8.

22. Jain $\mathrm{P}$, Marchant $\mathrm{T}$, Green $\mathrm{M}$, et al. Inter-fraction motion and dosimetric consequences during breast intensity-modulated radiotherapy (IMRT). Radiother Oncol. 2009;90:93-8.

23. van Mourik A, van Kranen S, den Hollander S, et al. Effects of setup errors and shape changes on breast radiotherapy. Int J Radiat Oncol Biol Phys. 2011:79:1557-64.

24. Ratosa I, Jenko A, Oblak I. Breast size impact on adjuvant radiotherapy adverse effects and dose parameters in treatment planning. Radiol Oncol. 2018;52(3):233-44.

25. Guo B, Shah CS, Magnelli A, et al. Surface Guided Radiation Therapy (SGRT): The Sensitivity of the Region of Interest (ROI) Selection on the Translational and Rotational Accuracy for Whole Breast Irradiation. Int J Radiat Oncol Biol Phys. 2011:2017(99):E666-7.

\section{Publisher's Note}

Springer Nature remains neutral with regard to jurisdictional claims in published maps and institutional affiliations. 